\title{
Dark Matter Candidates
}

\author{
Edward A. Baltz \\ KIPAC, Stanford University, P.O. Box 20450, MS 29, Stanford, CA 94309, USA
}

\begin{abstract}
It is now widely accepted that most of mass-energy in the universe is unobserved except by its gravitational effects. Baryons make only about $4 \%$ of the total, with "dark matter" making up about $23 \%$ and the "dark energy" responsible for the accelerated expansion of the universe making up the remainder. We focus on the dark matter, which is the primary constituent of galaxies. We outline the observed properties of this material, enumerating some candidates covering 90 orders of magnitude in mass. Finally, we argue that the weak scale $(100 \mathrm{GeV})$ is relevant to new physics, including the dark matter problem.
\end{abstract}

\section{THE DARK MATTER PROBLEM}

Cosmological parameter estimation, based on cosmic microwave background anisotropies measured by the WMAP satellite [1] and based on the power spectrum of galaxy density fluctuations measured by the SDSS collaboration [2] indicates that most of matter in the universe is unobserved save by gravity. Approximately $4 \%$ of the cosmological energy density is accounted for by baryons, $23 \%$ by the dark matter discussed in this lecture, with the remainder being the "dark energy" responsible for the observed accelerated expansion of the universe. The dark matter is the primary component of cosmological structures at the dwarf galaxy scale and larger. The dark matter and dark energy are of unknown composition. In this lecture we discuss the physics of dark matter, and enumerate a list of candidates, not meant to be exhaustive.

\section{DARK MATTER PROPERTIES}

In this section we enumerate five basic properties that dark matter is observed to have. The first three (that it must be non-radiating, approximately collisionless and non-relativistic) do not otherwise place any positive constraints on the space of possibilities, while the last two (that it must be fluid and classical) place upper and lower bounds respectively on the mass of the particles.

\subsection{Optically Dark (Dissipationless)}

Dark matter is not observed to shine, thus the dark matter particles must have very weak electromagnetic interactions. Either the electric charge and electric and magnetic dipole moments must vanish (or be very small), or the particles must be very heavy. For a review of constraints on dark matter from background light across the electromagnetic spectrum, see Ref. [3]. An important consequence of this is that the dark matter can not cool by radiating photons, and thus will not collapse to the center of galaxies as the baryons do, by radiating their energy away electromagnetically. In other words, the dark matter is very nearly dissipationless.

\subsection{Collisionless}

In addition to not interacting with "light" matter (to ensure that energy is not dissipated), the dark matter must be nearly collisionless as well. Even if the dark matter can not radiate energy, collisions will serve to make halos round, in contrast to data that overwhelmingly indicates triaxiality, e.g. in clusters [4]. The limit of this constraint is that there can be dark matter self interactions that are important at high densities and short distance scales, which may serve to erase small scale structure in galaxy halos, and erase the density cusps expected at the cores of galaxies. These effects appear for interaction cross sections of $\sigma \sim(m / \mathrm{GeV})(\lambda / \mathrm{Mpc})^{-1}$ barns [5], where $\lambda$ is the mean free 
path of the particles in typical halos. Essentially, this order of magnitude is indicative of hadrons, and there are a number of possibilities.

\subsection{Cold}

In the early 1980's it was noted that "cold" dark matter explained the observed properties of galaxies quite well [6]. The measured two point correlation function of galaxies indicates that there is a large amount of power on small scales. If the dark matter particles have significant velocities, then the small scale power is erased. For this not to occur, the particles must be sufficiently non-relativistic at the epoch of matter-radiation equality, when the temperature of the universe was roughly $1 \mathrm{eV}$ [7]. In practice, the constraint is that a particle species in thermal equilibrium must have a mass larger than about $1 \mathrm{keV}$ [8]. Of course, a non-thermal species can have a smaller mass. Dark matter that is not utterly cold may in fact ameliorate some of the difficulties of the cold dark matter model [9]; some suppression of small scale density fluctuations may be favored.

\subsection{Fluid}

The dark matter must be sufficiently fine on galaxy scales such that the discreteness is not detectable as yet. There are two basic effects to be concerned with. The first is that the granularity of dark matter provides a time dependent gravitational potential, which may disrupt bound systems. For particles of $10^{6} M_{\odot}$, this effect would heat the galactic disk to observable levels, while globular clusters would be disrupted for smaller masses, around $10^{3} M_{\odot}$ [10]. Second, the discreteness of dark matter introduces Poisson noise in the power spectrum of density fluctuations, which conflicts with observations of the Ly $\alpha$ forest if the particles are more massive than roughly $m \gtrsim 10^{4} M_{\odot}[11]$. We thus find that an upper bound on the mass of particles of roughly $m \lesssim 10^{3-4} M_{\odot} \sim 10^{70-71} \mathrm{eV}$.

Gravitational microlensing places further constraints on dark matter in the solar mass range. Searches for lenses by the MACHO and EROS collaborations have failed to find enough microlensing events to explain the full dark halo of the Milky Way [12], though MACHO reports that $20 \%$ of the Milky Way halo consists of objects of roughly $0.3 M_{\odot}$. The constraints are that objects between $10^{-7} M_{\odot}$ and $10 M_{\odot}$ do not make up more than about $20 \%$ of the dark matter in the Milky Way halo. Microlensing surveys are not able to place constraints much outside this mass range due to the limitations of microlensing event timescales.

\subsection{Classical}

Dark matter must behave sufficiently classically to be confined on galaxy scales. Bounds can be placed on the masses of both bosons and fermions, based only on the observed properties of galaxies: namely galaxy densities must reach of order $\mathrm{GeV} \mathrm{cm}{ }^{-3}$, their velocity dispersions are of order $100 \mathrm{~km} \mathrm{~s}^{-1}$, and their sizes are of order kpc.

\subsubsection{Bosons}

If dark matter consists of bosons, their quantum nature is manifest only if their mass is exceedingly small. In order to form galaxies, dark matter particles must be confined on kpc scales. With typical galactic velocities, the de Broglie wavelength of the particles is $\lambda \sim(\mathrm{eV} / \mathrm{m}) \mathrm{mm}$. Setting this wavelength to $1 \mathrm{kpc}$, we find that particles with mass $m \sim 10^{-22} \mathrm{eV}$ are barely confined. This mass denotes the lower end of the possible range for dark matter. In fact it was proposed as a mechanism to erase the small scale power in the galaxy power spectrum [13], called "fuzzy" cold dark matter. This also disallows the central density cusp found in numerical simulations of galactic dark halos.

\subsubsection{Fermions}

The lower bound on the mass of a fermionic dark matter particle is much more stringent than that for bosons. As first pointed out in Ref. [14], the phase space density of fermions has a maximum value $f=g h^{-3}$, where $g$ is the number of internal degrees of freedom, and in fact for a relativistic gas of fermions in thermal equilibrium it is 
half this value. In a galactic halo, the maximum phase space density assuming a Maxwellian velocity distribution is simply $f=\rho /\left[m^{4}\left(2 \pi \sigma^{2}\right)^{3 / 2}\right]$. Pauli blocking then enforces $m^{4}>\rho h^{3} /\left[g\left(2 \pi \sigma^{2}\right)^{3 / 2}\right]$. Taking a Milky Way type galaxy, where $\rho>1 \mathrm{GeV} \mathrm{cm}{ }^{-3}$ is required in the center and $\sigma=150 \mathrm{~km} \mathrm{~s}^{-1}$, and assuming $g=2$, we find $m \gtrsim 25 \mathrm{eV}$ for fermions.

\section{COSMOLOGICAL RELIC DENSITY OF PARTICLES}

The dark matter problem is a significant challenge for the particle physics community in that any candidate must satisfy stringent constraints. From a particle physics point of view, the properties discussed in section 2 are not hard to arrange, but two implicit properties are: namely that the candidate have a lifetime much longer than the Hubble time $\sim 10 \mathrm{Gyr}$, and also that its cosmological density is compatible with the observed dark matter density. The

current measured value of the dark matter density is $\Omega_{\mathrm{CDM}} h^{2}=0.135_{-0.009}^{+0.008}$ [1], where $\Omega$ is the density relative to the critical density $\rho_{c}=3 H_{0}^{2} /(8 \pi G)$, and $h\left(=0.71_{-0.03}^{+0.04}\right)$ is the scaled Hubble constant $H_{0}=100 h \mathrm{~km} \mathrm{~s}^{-1} \mathrm{Mpc}^{-1}$. In particle physics units, $\rho_{c} \approx 10.5 h^{2} \mathrm{keV} \mathrm{cm}^{-3}$. Accounting for the possibility of a significant neutrino density (see section 4.4), we take a $95 \%$ confidence region $0.087<\Omega_{\mathrm{CDM}} h^{2}<0.129$.

The simplest possibility would be for a candidate to have the correct density from thermal processes alone. Computing the relic density proceeds as follows [15]. The species is assumed to be in thermal equilibrium at early enough times, i.e. when the universe was essentially a radiation dominated plasma. The production and annihilation rates are tracked as the universe expands, and when these rates become longer than the (current) Hubble time, the species is said to be "frozen out" with a comoving density that no longer changes. This statement is expressed in the Boltzmann equation,

$$
\frac{d n}{d t}+3 H n=-\langle\sigma v\rangle\left(n^{2}-n_{\mathrm{eq}}^{2}\right),
$$

where $n$ and $n_{\text {eq }}$ are the particle density and its equilibrium value, $H$ is the Hubble parameter and $\langle\sigma v\rangle$ is annihilation cross section, appropriately thermally averaged [16]. We immediately notice that in the absence of interactions, the number density evolves exactly as expected, $n \propto a^{-3}$, where $a$ is the cosmic scale factor and $H=\dot{a} / a$. The relativistic gas has a temperature evolving as $T \propto a^{-1}$, thus $n \propto T^{3}$. The details of freeze out now depend on whether the relic is relativistic or not.

\subsection{Relativistic Thermal Freeze-out}

For relativistic particles, $n_{\mathrm{eq}} \propto T^{3}$, thus a species that has frozen out will still track the equilibrium density. The relic density of such particles is thus insensitive to the details of freeze-out. The only relevant parameter is the effective number of degrees of freedom at freeze-out, $g_{\star}$. This can change as a function of temperature, thus the density of a frozen out species need not track its equilibrium density, but the scaling with temperature remains as long as the particles are relativistic. The well known example of neutrinos freeze out with $g_{\star} \approx 10.75$, prior to the freeze-out of electrons and positrons (which lowers $g_{\star}$ ). Thus the (massless) neutrino temperature is lower than the photon temperature in the present universe. The relic density of any relativistic species is given by

$$
\Omega h^{2} \approx \frac{m}{100 \mathrm{eV}}\left(\frac{g_{\star}}{10}\right)^{-1} .
$$

\subsection{Non-Relativistic Thermal Freeze-out}

Non relativistic particles have an exponentially suppressed equilibrium density, $n_{\text {eq }} \propto(m T)^{3 / 2} \exp (-m / T)$. A frozen out species would thus have a density much larger than its equilibrium density. We can simply derive the scaling of relic density with annihilation cross section as follows. In a radiation dominated universe, $H \propto T^{2}$ (since $H^{2} \propto \rho$ according to the Friedman equation). The freeze-out condition can then be expressed simply as $H\left(T_{\mathrm{fr}}\right) \sim \sigma n_{\mathrm{eq}}\left(T_{\mathrm{fr}}\right)$. 
We find that $T_{\text {fr }} \propto m$, with only logarithmic corrections (this is due to the exponential Boltzmann factor). We can then derive the current mass density as $\rho \sim m n_{\mathrm{eq}}\left(T_{\mathrm{fr}}\right)\left(T / T_{\mathrm{fr}}\right)^{3} \sim T^{3}\left(m / T_{\mathrm{fr}}\right) / \sigma$. Thus we find the crucial result that $\rho \propto 1 / \sigma$, with only logarithmic dependence on mass since $m / T_{\text {fr }}$ is roughly constant. This means that smaller annihilation cross sections yield larger relic densities, which makes sense, as less efficient annihilations should allow more particles to survive. Taking the simple scaling that $\sigma \propto m^{-2}$, we find the approximate relic density

$$
\Omega h^{2} \sim\left(\frac{m}{\mathrm{TeV}}\right)^{2}
$$

This is a very interesting result in that a stable particle at the weak interaction scale of several hundred GeV would give the proper relic density to be dark matter. Any Weakly Interacting Massive Particle (WIMP) might thus be a compelling dark matter candidate.

\subsection{Pedagogical Example}

We can illustrate both the relativistic and non-relativistic freeze-out regimes with a single toy model as follows. Taking a Dirac fermion annihilating through a wide $Z^{\prime}$ boson, the annihilation cross section is

$$
\sigma v=\left(\frac{g^{4}}{64 \pi \cos ^{4} \theta_{W}}\right) \frac{m^{2}}{\left(s-m_{Z^{\prime}}^{2}\right)^{2}+m_{Z^{\prime}}^{4}} .
$$

In figure 1, we plot the relic density of this particle for a wide range of masses, as calculated by the DarkSUSY code [17]. Three regimes are evident. For masses below $1 \mathrm{MeV}$, the freeze out is relativistic, and the relic density is proportional to mass. Above $1 \mathrm{MeV}$, but below $m_{Z^{\prime}}$ the interaction is like low energy weak interactions, with $\sigma \propto E^{2}=m^{2}$, thus the relic density is proportional to the inverse square of the mass. For masses larger than the $Z^{\prime}$, the usual behavior of $\sigma \propto m^{-2}$ is recovered, with relic density proportional to the square of the mass. In a parallel set of three regimes, the boundaries between hot, warm, and cold dark matter are approximately illustrated. Comparing with the known value of the relic density, the hot and cold possibilities appear at $10 \mathrm{eV}$ and $1 \mathrm{TeV}$ respectively.

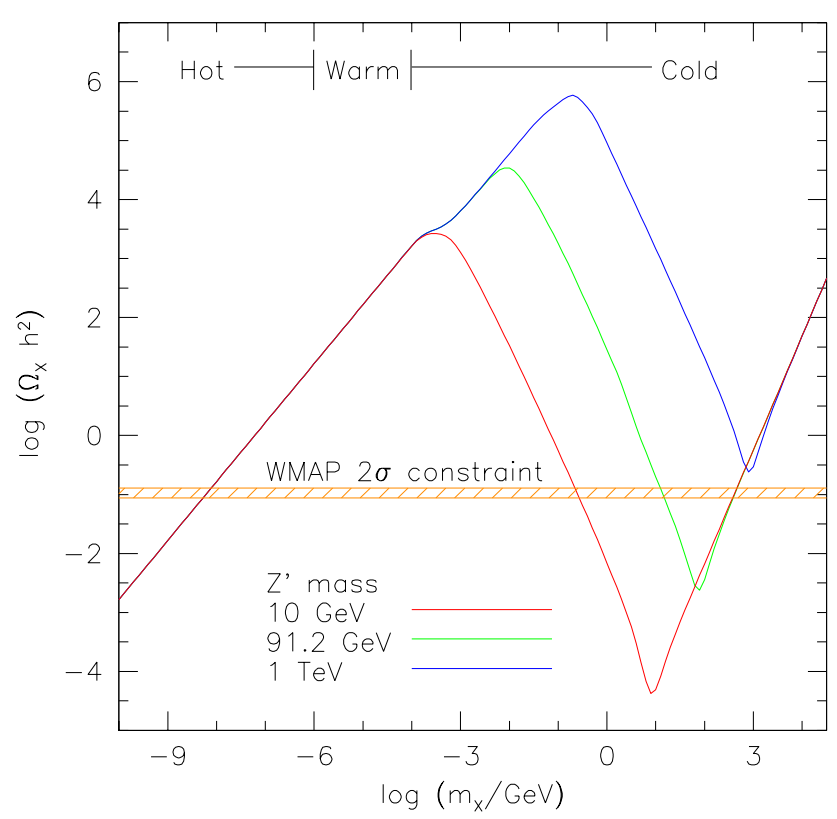

Figure 1: Relic density of a species freezing out in thermal equilibrium. The interaction cross section is of weak interaction strength. For masses below $\sim \mathrm{MeV}$, the particles are relativistic at freeze-out, above this mass they are non-relativistic. Above a mass of $\sim 100 \mathrm{GeV}$, the interactions look "electromagnetic" as the gauge boson becomes light relative to the particle mass. The three regimes of cold, warm, and hot dark matter are illustrated, along with the WMAP $2 \sigma$ constraint on dark matter density. 


\section{DARK MATTER CANDIDATES}

In this section we outline some of the proposals for dark matter. While this list is by no means exhaustive, we will attempt to cover the range of possibilities that have been considered at least qualitatively. We will proceed very roughly from the smallest mass candidates at $10^{-22} \mathrm{eV}$ (the fuzzy cold dark matter discussed previously) to the largest at $10^{6} M_{\odot} \approx 10^{72} \mathrm{eV}$. This range covers more than 90 orders of magnitude in mass; unfortunately observations do not place stronger bounds on possible candidates. However, the weak scale $(100 \mathrm{GeV})$ is quite interesting for the dark matter problem, as we will describe.

\section{1. "Fuzzy" Cold Dark Matter}

This model was discussed in section 2.5.1.

\subsection{Chaplygin Gas}

The Chaplygin gas is an attempt to unify dark matter and dark energy in a single fluid with an equation of state $p=-A / \rho$. Generalizations of this have also been considered [18]. This type of fluid can arise in certain string-inspired models involving $d$-branes. The transition between the dust-like universe at moderate redshift to the accelerating universe today occurs naturally in these models.

\subsection{Axions}

Strong interactions naively violate $C P$ with order unity strength (the coefficient $\theta$ of the $F \tilde{F}$ term in the QCD Lagrangian is not obviously small), while experimentally the violation must be smaller than $\theta \sim 10^{-9}$. Promoting the parameter to a dynamical field solves this problem, at the expense of a new light pseudoscalar boson, the axion [19]. The cosmology and astrophysics of these particles has been studied at length, beginning with Ref. [20] and reviewed in Refs. [21]. To summarize, there is an allowed mass window of interest to dark matter, with $\mu \mathrm{eV} \lesssim m \lesssim$ meV. The lower bound comes the fact that the relic density of axions $\Omega_{a} h^{2} \simeq\left(4 \mu \mathrm{eV} / m_{a}\right)^{7 / 6}\left(200 \mathrm{MeV} / \Lambda_{\mathrm{QCD}}\right)^{3 / 4}$, while the upper bound comes from the fact that the axion interactions must be weak enough that stars cool via photons and not axions. Axion production is non-thermal, thus they are cold, even though their masses are far below the $\sim \mathrm{keV}$ limit for warm dark matter. Early on it was pointed out that axions could be detected by resonant axion-photon conversion in a magnetic field [22], an experimental program that has been undertaken with some success [23]. Axion dark matter is particularly interesting from an experimental point of view in that the velocity distribution of the local dark can be measured with high accuracy. A record of the process of virialization would be evident, of considerable interest to studies of galaxy formation.

\subsection{Neutrinos}

We now know that neutrinos are one component of the cosmological dark matter. Neutrino oscillation experiments, both for atmospheric neutrinos [24] and for solar neutrinos [25], have now conclusively shown that at least two of the neutrinos in the Standard Model are massive. Measurements of the endpoint spectrum of tritium beta decay place a $95 \%$ confidence upper bound on the electron neutrino mass, $m_{\nu_{e}} \lesssim 2.5 \mathrm{eV}$ [26], thus bounding all three neutrino species as the mass splittings measured by the oscillation experiments are both much smaller than this. The cosmological density of light neutrinos $\left(m_{\nu} \lesssim \mathrm{MeV}\right)$ is known to be $\Omega_{\nu} h^{2}=\sum m_{\nu} /(94.0 \mathrm{eV})$ [27]. The WMAP bound [1] on this quantity is $\Omega_{\nu} h^{2}<0.0076 \rightarrow \sum m_{\nu} \lesssim 0.7 \mathrm{eV}$, thus the cosmological bound is more stringent than the laboratory one $\left(\sum m_{\nu} \lesssim 7.5 \mathrm{eV}\right)$. The measured oscillation parameters enforce that $\sum m_{\nu} \gtrsim 50 \mathrm{meV}$, and thus $0.0005<\Omega_{\nu} h^{2}<0.0076$, a known constituent of dark matter as even the bottom of this mass range would be non-relativistic today. However, no fermion in this mass range can be a significant constituent of galaxies due simply 
to the Pauli exclusion principle discussed above. In addition to not being abundant enough, these Standard Model neutrinos are "hot" and thus unsuitable to explain the properties of galaxies.

Very massive neutrinos have also been considered as dark matter candidates, first in Ref. [28]. We now know that none of the Standard Model neutrinos are suitable, but their right-handed counterparts might be acceptable. Active neutrinos in a 4th generation would be acceptable except for the fact that they would have been detected in sensitive underground experiments by now.

\subsection{MeV Dark Matter}

The INTEGRAL satellite has observed an excess of $511 \mathrm{keV}$ positron annihilation emission toward the galactic center, which is difficult to reconcile with astrophysical sources. It has been suggested that this emission might be due to $e^{+} e^{-}$pairs that are the annihilation products of dark matter particles in the MeV range [30]. Their mass must be low to exclude hadronic final states, which would overproduce photons from e.g. $\pi^{0}$ decays, and also so that the leptons are not so energetic that they escape the galactic center region. However, the particle physics motivation for this scenario is weaker than for other candidates.

\subsection{Supersymmetry}

Our current understanding of physics includes no fundamental relationship between bosons and fermions. Supersymmetry was proposed as just such a relationship, first on the 2-d worldsheet for string theory [31], and slightly later for standard 4-d spacetime [32]. Supersymmetric theories put bosons and fermions in common multiplets; for most such theories then the corresponding states must have similar masses. It is thus obvious that if supersymmetry is a valid symmetry, it is badly broken in the low energy world in which we live. These theories admit the so-called non-renormalization theorem, which remains valid if the supersymmetry is broken "softly". Very roughly stated, the only infinite renormalizations are of wavefunction type, and those are only logarithmically divergent. The quadratically divergent corrections in the Standard Model are absent.

The Minimal Supersymmetric Standard Model (MSSM, for early reviews see Refs. [33]) was proposed as a model with broken supersymmetry. Each Standard Model state has a superpartner with spin differing by $\hbar / 2$ : matter fermions (quarks, leptons) have scalar partners, gauge bosons have spin 1/2 partners, and Higgs bosons (two $S U(2)$ doublets are required) also have spin $1 / 2$ partners. The soft supersymmetry breaking in this model allows for the masses of superpartners to differ from their Standard Model counterparts. The most general gauge invariant Lagrangian involving these fields includes terms that violate either baryon or lepton number, e.g. a term allowing scalar up-type squarks to decay directly to a down quark and charged lepton. If both baryon and lepton number violating interactions are included, proton decay occurs at the weak scale. The usual solution is to impose R-parity conservation, where superpartners have negative R-parity and thus must interact in pairs. This means that the lightest superpartner (LSP) is absolutely stable. Early on, it was realized that the LSP (first considered to be the gravitino) was thus a dark matter candidate [34].

\subsubsection{Gravitinos}

The superpartner of of the graviton, the spin $3 / 2$ gravitino, was the first SUSY particle considered for the dark matter problem [34]. In models where the gravitino is the LSP, it is often quite light (keV), and would thus be warm dark matter. In cosmology, the overproduction of gravitinos is somewhat problematic, though not insurmountably so [35]. Gravitinos at the weak scale, whose relic density would be obtained through the decays of the next lightest superpartner, are also an interesting possibility [36].

\subsubsection{Neutralinos}

The favored supersymmetric dark matter candidate is the lightest neutralino; these are the four spin 1/2 Majorana fermion superpartners of the neutral gauge and Higgs bosons (usually denoted $\chi_{1-4}^{0}$ ) [37]. Similarly, there are two 
charged Dirac fermion superpartners of charged gauge and Higgs bosons, the charginos $\chi_{1-2}^{ \pm}$. The successes of supersymmetric models depend on the fact that the gauginos have weak-scale masses, thus the proper relic density for any stable states comes essentially for free. We focus on neutralinos as dark matter in section 5.

\subsubsection{Sneutrinos}

The scalar partners of the neutrinos are possible dark matter candidates, but they are disfavored for two reasons. First, they annihilate quite efficiently, requiring masses above $500 \mathrm{GeV}$ to provide a relic density consistent with dark matter. Second, their elastic scattering cross sections on nuclei are quite large (on the order of femtobarns), to the point that they would be easily detectable in current experiments [38].

\subsubsection{Axinos}

If the axions proposed to solve the strong $C P$ problem exist (see section 4.3) and in addition supersymmetry is valid, the axion will naturally have a spin $1 / 2$ partner, the axino. Depending on the conditions in the early universe, these might be either warm or cold dark matter [39].

\subsubsection{Q-balls}

Supersymmetric theories typically permit non-topological solitons, dubbed Q-balls. These carry baryon and/or lepton number, and can be absolutely stable if large enough. They are an interesting dark matter candidate, and may even have relatively strong self-interactions at the level discussed in section 2.2 [40].

\subsubsection{Split SUSY}

Recently it has been noted that the successes of supersymmetric models hinge on the fact that the gauginos have masses at the weak scale, while having the scalar superpartners at the weak scale is somewhat problematic. If the Higgs fine-tuning problem is simply ignored (as the cosmological constant fine-tuning often is), the scalar superpartners can be made very heavy, keeping light gauginos and Higgsinos [41]. This scenario has an interesting phenomenology, both for collider signatures and for dark matter [42].

\subsection{Universal Extra Dimensions}

If our four-dimensional spacetime is embedded in a higher dimensional space, excitations of Standard Model states along the orthogonal dimensions (called Kaluza-Klein excitations) may be viable dark matter candidates [43]. For example, the first excitation of the $B$ boson (associated with the $U(1)_{Y}$ hypercharge gauge group of the Standard Model) has been considered. This is similar to supersymmetry in that known particles have partners, but the partners' spin does not differ. This scenario has been dubbed "bosonic supersymmetry". The stability of the lightest of the KK excitations can be arranged by a parity symmetry, and masses around $1 \mathrm{TeV}$ provide reasonable relic densities from thermal freeze-out, the same as with supersymmetric models.

\subsection{Branons}

String theory naturally contains objects of many different dimensions, called branes. These would naturally have fluctuations characterizable as particles, the so-called branons. These fluctuations can be made into suitable cold dark matter candidates, both thermal and non-thermal [44]. 


\subsection{Mirror Matter}

The (modern) concept of a mirror world is an old one, extending back to the non-conservation of parity in weak interactions. In a mirror scenario, the dark matter could just be ordinary matter in the mirror world, where the only communication is gravitational. This scenario can be constructed in a braneworld context, where our universe and a mirror universe are two branes in a higher dimensional space. For more complete discussions of this scenario, see e.g. [45].

\subsection{WIMPzillas}

At the end of inflation, gravitational interactions alone can produce copious particles. For mass scales of $10^{13} \mathrm{GeV}$, these particles, if stable, could even account for the dark matter [46]. In addition, such particles might decay with a lifetime much longer than the age of the universe, providing a source of ultra high energy cosmic rays - this is the so-called "top-down" scenario for UHECR production.

\subsection{Primordial Black Holes}

Under the right conditions, primordial black holes can form in the early universe, e.g. see Ref. [47]. Production is enhanced during periods where the equation of state softens $(p<\rho / 3)$, such as during a first order phase transition. This is easy to understand: if the pressure support lessens, objects collapse more easily. The last such phase transition in the universe is the quark-hadron phase transition, at a temperature $T \sim 100 \mathrm{MeV}$. The mass contained in the horizon at this epoch is very roughly a solar mass.

\section{FOCUS ON NEUTRALINOS}

The lightest neutralino is the dark matter candidate that has endured the most scrutiny. In this section we elaborate on the discussion of the MSSM, and discuss the prospects for discovering neutralinos astrophysically. The MSSM starts with the Standard Model field content, adds one additional $S U(2)$ doublet of Higgs bosons (with the opposite $U(1)_{Y}$ charge), and then adds the superpartners of each of these states. The Lagrangian includes all SUSY conserving terms that respect R-parity, and SUSY-breaking soft terms. More than 100 new parameters are required. One parameter of the Standard Model is actually removed, the quartic self-coupling of the Higgs boson. These couplings appear in the MSSM, but with the quartic coupling $\lambda_{\mathrm{SM}}$ replaced by terms in the gauge couplings $g^{2}$ and $g^{\prime 2}$. Clearly, a theory with $100+$ free parameters is not very predictive. To make some connection to reality, a simpler model is required.

\subsection{Minimal Supergravity}

The best studied model of low-energy supersymmetry uses gravitational effects to break supersymmetry, called Minimal Supergravity (mSUGRA) [48]. The model has four parameters, plus one sign, beyond the Standard Model ones (and again, $\lambda_{\mathrm{SM}}$ is absent). These continuous parameters are $m_{0}$, the universal scalar mass at the GUT scale, $m_{1 / 2}$, the universal GUT-scale gaugino mass, $A_{0}$, the GUT-scale trilinear coupling, and $\tan \beta$, the weak-scale ratio of the vacuum expectation values of the two scalar neutral Higgs fields. The Higgsino mass parameter $|\mu|^{2}$ is determined by enforced radiative electroweak symmetry breaking, but the phase of $\mu$ is undetermined: it is chosen to be \pm 1 to conserve $C P$.

In studying this model, a set of parameters is evolved according to the renormalization group equations (RGEs), checking that the gauge couplings unify, and electroweak symmetry breaking occurs. There are a number of publicly available software packages that solve RGEs: we use ISAJET [49]. Further tests against accelerator data (e.g. 
sparticle masses, $\mathrm{BR}(b \rightarrow s \gamma)$, etc.) are performed. Finally, parameter sets where the LSP is not a neutralino are discarded.

We can roughly understand the weak-scale parameters as follows. As the gaugino masses are unified at the GUT scale, their weak-scale masses are related to the ratios of the gauge coupling constants at the weak scale: strong, weak, hypercharge gaugino masses have $M_{3}: M_{2}: M_{1} \sim 6: 2: 1$, namely the B-ino is the lightest gaugino. Gauge couplings run scalar masses higher, while Yukawa couplings run scalar masses lower: squarks are heavy, sleptons are light, with $\tilde{u}_{L}$ usually heaviest and $\tilde{\tau}_{R}$ usually lightest.

To connect these models to cosmology, we must calculate the relic density of neutralinos. We use the publicly available DarkSUSY code for this purpose [17]. The code actually calculates the relic density of all neutralinos, charginos and sfermions; if the mass splittings are small, the "coannihilation" effects can be crucial. Given the current experimental limits, three regions of mSUGRA parameter space stand out as giving a relic density compatible with cosmology: stau coannihilations, where the $\chi_{1}^{0}-\tilde{\tau}_{R}$ splitting is small, the focus point region where the $\chi_{1}^{0}-\chi_{2}^{0}-\chi_{1}^{ \pm}$ splittings are small, and the region (at large $\tan \beta$ ) where the annihilation through the pseudoscalar Higgs $A^{0}$ is resonant, $\chi \chi \rightarrow A^{0} \rightarrow f \bar{f}$.

\subsection{MCMC Scanning}

The regions of mSUGRA parameter space compatible with cosmology are quite narrow, and difficult to find in general. Grid searches of the parameter space are $\sim 1 \%$ efficient at finding models passing the $95 \%$ confidence cut mentioned in section 3 . However, we can use the relic density of models to guide the search through parameter space, using the so-called Markov Chain Monte Carlo (MCMC) [50]. The basic idea is to use a likelihood function $\mathcal{L}=e^{-\chi^{2} / 2}$ (here taken as the WMAP likelihood function for relic density) calculated at the current point in parameter space. The likelihood is calculated at a proposed point, and the "chain" advances if the likelihood is higher. With lower likelihood, the chain advances with probability $\mathcal{L}_{\text {new }} / \mathcal{L}_{\text {old }}$. In this way chains of models can be constructed that efficiently sample the interesting regions. A 2 dimensional example is illustrated in figure 2 . In fact we have explored the full 4 dimensional mSUGRA parameter space, finding efficiencies with MCMC of 20\%-25\% (a huge improvement). We scanned 2.4 million models in total, finding 500 thousand passing the WMAP cut.

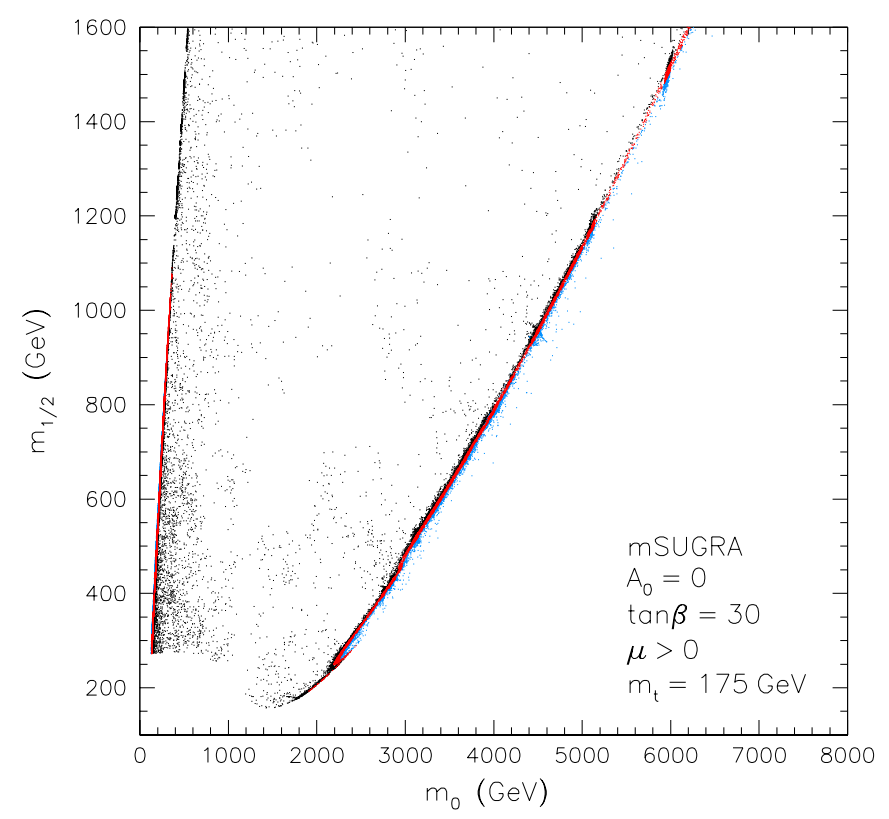

Figure 2: MCMC scan in 2 dimensions. We have fixed $A_{0}=0$, $\tan \beta=30, m_{t}=175 \mathrm{GeV}$. Black points illustrate models with relic density too high, red points pass the $95 \%$ confidence WMAP cut, and blue points fall below (thus they are acceptable, but not as a full solution for the dark matter problem. The stau coannihilation region clearly appears at the left edge, while the focus point region (chargino coannihilation) is clear at the right edge. At larger $\tan \beta$, the Higgs resonance region would appear. The stau coannihilation region ends at $m_{1 / 2} \sim 1100 \mathrm{GeV}$, while the focus point extends at least to $m_{1 / 2} \sim 20 \mathrm{TeV}(10 \mathrm{TeV}$ neutralinos). 


\subsection{Direct Detection}

On of the most promising experimental techniques for discovering neutralinos is "direct detection". The idea is that the neutralino will scatter on a nucleus, depositing energy that can be detected in a number of ways [51]. The scatterings are very rare (the current limits are of the order of $\sigma \sim 10^{-42} \mathrm{~cm}^{2}$ ), and they deposit very little energy (typically $\sim 10 \mathrm{keV}$ ), so the experimental challenge is considerable. In figure 3, we plot all of the mSUGRA models consistent with solving the dark matter problem. The axes are neutralino mass and spin-independent neutralinonucleon elastic scattering cross section. The spin independent scattering amplitudes essentially couple to the mass of the nucleon, and add in quadrature due to coherent effects (the $10 \mathrm{keV}$ exchanged is long wavelength compared to a nucleus). Current experimental efforts are just starting to probe the mSUGRA region, with several orders of magnitude improvement being quite attainable in the near future. Spin-dependent interactions are also present, but they are experimentally more difficult to explore, requiring odd-A targets (though the cross sections can be quite large). In figure 4 we plot the spin-dependent cross sections on protons and neutrons.

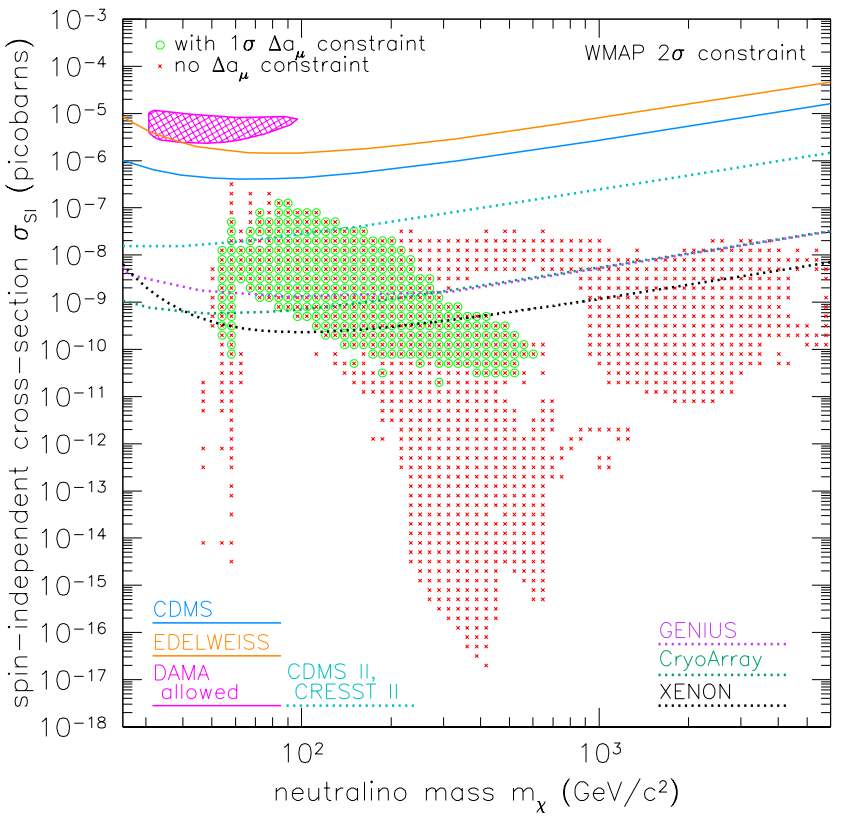

Figure 3: Direct detection of neutralinos. The spinindependent elastic scattering cross section of neutralinos on protons is plotted against neutralino mass. Red crosses indicate the mSUGRA parameter space, while green circles indicate those models that could make a significant contribution to the $g-2$ of the muon, which may be experimentally favored [52]. Current limits from the EDELWEISS and CDMS experiments are plotted [53], along with the projected sensitivities of several proposed experiments [54]. The region consistent with the DAMA annual modulation claim is illustrated as well [55]; this region is inconsistent with the negative results of other experiments.

\subsection{Indirect Detection}

Here, we will briefly mention the indirect techniques for neutralino searches. The first relies on the fact that massive objects such as the Earth or Sun will collect neutralinos due to elastic scattering into bound gravitational orbits. The particles thereafter become concentrated near the center of such objects, and annihilate more rapidly. The neutrinos from the annihilation products can be searched for in neutrino telescopes [56].

Neutralino annihilations in the galactic halo proceed at a very small rate, but the annihilation products are very energetic and might thus be detectable. These are (focusing on antiparticles) gamma rays, antiprotons, positrons, and antideuterons. Monochromatic gamma rays are particularly interesting; the $\chi \chi \rightarrow \gamma \gamma, Z^{0} \gamma$ branching ratios are typically quite small, but the signal is unmistakable and has no astrophysical background. 



Figure 4: Direct detection of neutralinos. The spin-dependent elastic scattering cross sections of neutralinos on protons and neutrons are plotted against neutralino mass. Symbols are as in figure 3.

\section{CAN WE LIVE WITHOUT DARK MATTER?}

Observed matter can not account for the gravitational potentials of objects at the dwarf galaxy scale and larger, thus dark matter was hypothesized. An alternative possibility is that gravity weakens less quickly at large distances, or as proposed by Milgrom [57], at small acceleration scales. The latter idea is known as MOND, for MOdified Newtonian Dynamics. Basically, imposing a minimum acceleration scale $a_{0} \sim c H_{0}$ turns out to explain spiral galaxies quite well. If this MOND scale $a_{0}$ is allowed to run, clusters might be explained as well. Because gravitational lensing mass estimates agree with dynamical estimates, the MOND acceleration scale must apply to photons as well. A relativistic theory is needed in any case to construct a consistent cosmology. A number of attempts have been made, each with fatal inconsistencies [58]. Quite recently, Bekenstein has proposed a tensor-vector-scalar theory (TeVeS) that is successful in reproducing MOND in the proper limit even for photons, and respecting the classical tests of general relativity [59]. Whether a consistent cosmology can be constructed with the theory remains to be seen. Note that even this is a "dark matter" theory, in that the structure of galaxies is still explained by the introduction of new particles.

\section{DISCUSSION}

We are unable to escape the fact that new physics is required to explain the large scale structure of the universe. Measurements of the cosmological matter density, most recently by the WMAP satellite, are precise indications $(7 \%$ error, and fast improving) of something relating to this new physics. Identifying exactly what that something is is one of the chief challenges in physics in the next decade(s). 


\section{Acknowledgments}

We thank the organizers of the XXXII SLAC Summer Institute for the invitation to lecture on dark matter and for

putting on such an informative school. This work was supported by the U.S. Department of Energy under contract number DE-AC02-76SF00515.

\section{References}

[1] C. L. Bennett et al. [WMAP Collaboration], Astrophys. J. Suppl. 148, 1 (2003);

D. N. Spergel et al. [WMAP Collaboration], Astrophys. J. Suppl. 148, 175 (2003).

[2] K. Abazajian et al. [SDSS Collaboration], Astron. J. 126, 2081 (2003);

M. Tegmark et al. [SDSS Collaboration], Phys. Rev. D 69, 103501 (2004).

[3] J. M. Overduin and P. S. Wesson, Phys. Rept. 402, 267 (2004).

[4] J. J. Mohr, A. E. Evrard, D. G. Fabricant, and M. J. Geller, Astrophys. J. 447, 8 (1995).

[5] D. N. Spergel and P. J. Steinhardt, Phys. Rev. Lett. 84, 3760 (2000).

[6] P. J. E. Peebles, Astrophys. J. Lett. 263, L1 (1982);

G. R. Blumenthal, S. M. Faber, J. R. Primack and M. J. Rees, Nature 311, 517 (1984).

[7] J. R. Bond and A. S. Szalay, Astrophys. J. 274, 443 (1983);

J. M. Bardeen, J. R. Bond, N. Kaiser and A. S. Szalay, Astrophys. J. 304, 15 (1986).

[8] V. K. Narayanan, D. N. Spergel, R. Davé, C.-P. Ma, Astrophys. J. 543, L103 (2000).

[9] J. Sommer-Larsen, A. Dolgov, Astrophys. J. 551, 608 (2001);

P. Bode, J. Ostriker, N. Turok, Astrophys. J. 556, 93 (2001).

[10] C. G. Lacey and J. P. Ostriker, Astrophys. J. 299, 633 (1985);

B. Moore, Astrophys. J. 413, L93 (1993);

H. Rix and G. Lake, Astrophys. J. 417, L1 (1993).

[11] N. Afshordi, P. McDonald and D. N. Spergel, Astrophys. J. 594, L71 (2003).

[12] C. Alcock et al., Astrophys. J. 542, 281 (2000);

C. Afonso et al., Astron. Astrophys. 400, 951 (2003).

[13] W. Hu, R. Barkana and A. Gruzinov, Phys. Rev. Lett. 85, 1158 (2000).

[14] S. Tremaine and J. E. Gunn, Phys. Rev. Lett. 42, 407 (1979).

[15] e.g. see E. W. Kolb, M. S. Turner, The Early Universe, Addison-Wesley (1990).

[16] P. Gondolo and G. Gelmini, Nucl. Phys. B360, 145 (1991).

[17] P. Gondolo, J. Edsjo, P. Ullio, L. Bergstrom, M. Schelke and E. A. Baltz, JCAP 0407, 008 (2004).

[18] A. Y. Kamenshchik, U. Moschella and V. Pasquier, Phys. Lett. B 511, 265 (2001);

M. C. Bento, O. Bertolami and A. A. Sen, Phys. Rev. D 66, 043507 (2002).

[19] R. D. Peccei and H. R. Quinn, Phys. Rev. Lett. 38, 1440 (1977);

S. Weinberg, Phys. Rev. Lett. 40, 223 (1978);

F. Wilczek, Phys. Rev. Lett. 40, 279 (1978).

[20] P. Sikivie, Phys. Rev. Lett. 48, 1156 (1982);

J. Preskill, M. B. Wise and F. Wilczek, Phys. Lett. B 120, 127 (1983);

L. F. Abbott and P. Sikivie, Phys. Lett. B 120, 133 (1983);

M. Dine and W. Fischler, Phys. Lett. B 120, 137 (1983).

[21] M. S. Turner, Phys. Rept. 197, 6797 (1990);

G. G. Raffelt, Phys. Rept. 198, 1113 (1990).

[22] P. Sikivie, Phys. Rev. Lett. 51, 1415 (1983).

[23] For the most recent published results see S. J. Asztalos et al., Astrophys. J. 571, L27 (2002).

[24] Y. Fukuda et al. [Super-Kamiokande Collaboration], Phys. Rev. Lett. 81, 1562 (1998).

[25] Q. R. Ahmad et al. [SNO Collaboration], Phys. Rev. Lett. 89, 011301 (2002). 
[26] C. Weinheimer et al., Phys. Lett. B 460, 219 (1999);

V. M. Lobashev et al., Phys. Lett. B 460, 227 (1999).

[27] R. Cowsik and J. McClelland, Phys. Rev. Lett. 29, 669 (1972).

[28] B. W. Lee and S. Weinberg, Phys. Rev. Lett. 39, 165 (1977).

[29] J. Knodlseder et al., Astron. Astrophys., in press (arXiv:astro-ph/0309442);

P. Jean et al., Astron. Astrophys. 407, L55 (2003).

[30] C. Boehm, D. Hooper, J. Silk and M. Casse, Phys. Rev. Lett. 92, 101301 (2004).

[31] P. Ramond, Phys. Rev. D 3, 2415 (1971).

[32] Y. A. Golfand and E. P. Likhtman, JETP Lett. 13, 323 (1971);

J. Wess and B. Zumino, Phys. Lett. B 49, 52 (1974).

[33] H. P. Nilles, Phys. Rept. 110, 1 (1984);

H. E. Haber and G. L. Kane, Phys. Rept. 117, 75 (1985).

[34] H. Pagels and J. R. Primack, Phys. Rev. Lett. 48, 223 (1982).

[35] J. R. Ellis, A. D. Linde and D. V. Nanopoulos, Phys. Lett. B 118, 59 (1982);

T. Moroi, H. Murayama and M. Yamaguchi, Phys. Lett. B 303, 289 (1993).

M. Kawasaki and T. Moroi, Prog. Theor. Phys. 93, 879 (1995).

[36] J. L. Feng, A. Rajaraman and F. Takayama, Phys. Rev. Lett. 91, 011302 (2003);

J. L. Feng, A. Rajaraman and F. Takayama, Phys. Rev. D 68, 063504 (2003).

[37] S. Weinberg, Phys. Rev. Lett. 48, 1303 (1982);

H. Goldberg, Phys. Rev. Lett. 50, 1419 (1983);

reviewed in G. Jungman, M. Kamionkowski and K. Griest, Phys. Rept. 267, 195 (1996).

[38] J. S. Hagelin, G. L. Kane and S. Raby, Nucl. Phys. B 241, 638 (1984);

L. E. Ibanez, Phys. Lett. B 137, 160 (1984);

T. Falk, K. A. Olive and M. Srednicki, Phys. Lett. B 339, 248 (1994).

[39] J. E. Kim, A. Masiero and D. V. Nanopoulos, Phys. Lett. B 139, 346 (1984);

S. A. Bonometto, F. Gabbiani and A. Masiero, Phys. Lett. B 222, 433 (1989);

K. Rajagopal, M. S. Turner and F. Wilczek, Nucl. Phys. B 358, 447 (1991);

L. Covi, J. E. Kim and L. Roszkowski, Phys. Rev. Lett. 82, 4180 (1999).

[40] G. Rosen, J. Math. Phys. 9, 996 (1968);

R. Friedberg, T. D. Lee and A. Sirlin, Phys. Rev. D 13, 2739 (1976);

S. R. Coleman, Nucl. Phys. B 262, 263 (1985);

T. D. Lee and Y. Pang, Phys. Rept. 221, 251 (1992);

A. Kusenko, Phys. Lett. B 404, 285 (1997);

A. Kusenko, Phys. Lett. B 405, 108 (1997);

A. Kusenko and M. E. Shaposhnikov, Phys. Lett. B 418, 46 (1998);

A. Kusenko and P. J. Steinhardt, Phys. Rev. Lett. 87, 141301 (2001).

[41] J. D. Wells, arXiv:hep-ph/0306127;

N. Arkani-Hamed and S. Dimopoulos, arXiv:hep-th/0405159.

[42] G. F. Giudice and A. Romanino, Nucl. Phys. B 699, 65 (2004);

N. Arkani-Hamed, S. Dimopoulos, G. F. Giudice and A. Romanino, arXiv:hep-ph/0409232;

J. D. Wells, arXiv:hep-ph/0411041;

A. Pierce, Phys. Rev. D 70, 075006 (2004).

[43] I. Antoniadis, Phys. Lett. B 246, 377 (1990);

I. Antoniadis, K. Benakli and M. Quirós, Phys. Lett. B 331, 313 (1994);

H. C. Cheng, K. T. Matchev and M. Schmaltz, Phys. Rev. D 66, 056006 (2002);

G. Servant and T. M. P. Tait, Nucl. Phys. B 650, 391 (2003);

E. W. Kolb and R. Slansky, Phys. Lett. B 135, 378 (1984);

K. R. Dienes, E. Dudas and T. Gherghetta, Nucl. Phys. B 537, 47 (1999). 
[44] J. A. R. Cembranos, A. Dobado and A. L. Maroto, Phys. Rev. Lett. 90, 241301 (2003).

[45] T. D. Lee and C. N. Yang, Phys. Rev. 104, 254 (1956);

I. Yu. Kobzarev, L. B. Okun, and I. Ya Pomeranchuk, Yad. Fiz. 3, 1154 (1966);

E. Kolb, D. Seckel, and M. Turner, Nature 314, 415 (1985);

H. M. Hodges, Phys. Rev. D 47, 456 (1993);

Z. Berezhiani, D. Comelli and F. L. Villante, Phys. Lett. B 503, 362 (2001);

R. Foot, Acta Phys. Polon. B 32, 2253 (2001).

[46] D. J. H. Chung, E. W. Kolb and A. Riotto, Phys. Rev. Lett. 81, 4048 (1998);

D. J. H. Chung, E. W. Kolb and A. Riotto, Phys. Rev. D 59, 023501 (1999).

[47] Ya. B. Zel'dovich and I. D. Novikov, Sov. Astron. 10, 62 (1967);

S. Hawking, Mon. Not. Roy. Astron. Soc. 152, 75 (1971);

B. J. Carr, Astrophys. J. 201, 1 (1975);

K. Jedamzik, Phys. Rev. D 55, 5871 (1997).

[48] A. H. Chamseddine, R. Arnowitt and P. Nath, Phys. Rev. Lett. 49, 970 (1982);

R. Barbieri, S. Ferrara and C. A. Savoy, Phys. Lett. B 119, 343 (1982);

N. Ohta, Prog. Theor. Phys. 70, 542 (1983);

L. J. Hall, J. Lykken and S. Weinberg, Phys. Rev. D 27, 2359 (1983).

[49] H. Baer, F. E. Paige, S. D. Protopescu and X. Tata, arXiv:hep-ph/0312045.

[50] E. A. Baltz and P. Gondolo, JHEP 0410, 052 (2004).

[51] M. W. Goodman and E. Witten, Phys. Rev. D 31, 3059 (1985).

[52] G. W. Bennett, et al., Phys. Rev. Lett. 92, 161802 (2004);

M. Davier, S. Eidelman, A. Höcker and Z. Zhang, Eur. Phys. J. C 31, 503 (2003);

K. Hagiwara, A. D. Martin, D. Nomura and T. Teubner, Phys. Rev. D 69, 093003 (2004).

[53] A. Benoit et al. [EDELWEISS Collaboration], Phys. Lett. B 545, 43 (2002);

D. S. Akerib et al. [CDMS Collaboration], Phys. Rev. Lett. 93, 211301 (2004).

[54] CDMS II, http://cdms.berkeley.edu/

CRESST II, http://wwwvms .mppmu.mpg.de/cresst/

GENIUS, H. V. Klapdor-Kleingrothaus et al., in "Beyond the desert 1999," (IOP, 2000), p. 915;

CryoArray, R. W. Schnee, D. S. Akerib and R. J. Gaitskell, arXiv:astro-ph/0208326;

XENON, E. Aprile et al., arXiv:astro-ph/0207670, http://www.astro.columbia.edu/ $1 x e / X E N O N /$.

[55] R. Bernabei et al., Riv. Nuovo Cim. 26N1, 1 (2003).

[56] J. Silk, K. A. Olive and M. Srednicki, Phys. Rev. Lett. 55, 257 (1985);

K. Freese, Phys. Lett. B 167, 295 (1986);

L. M. Krauss, M. Srednicki and F. Wilcek, Phys. Rev. D 33, 2079 (1986).

[57] M. Milgrom, Astrophys. J. 270, 365 (1983);

M. Milgrom, Astrophys. J. 270, 371 (1983);

M. Milgrom, Astrophys. J. 270, 384 (1983).

[58] J. Bekenstein and M. Milgrom 1984, Astrophys. J. 286, 7 (1984);

J. D. Bekenstein, Phys. Lett. B 202, 497 (1988);

M. E. Soussa and R. P. Woodard, Class. Quant. Grav. 20, 2737 (2003).

[59] J. D. Bekenstein, Phys. Rev. D 70, 083509 (2004). 